\title{
A BILATERAL AND MULTI-ISSUE NEGOTIATION FRAMEWORK TO SUPPORT A SUPPLY CHAIN OF CONSTRUCTION INDUSTRY
}

\author{
Fernando Schramm ${ }^{1}$ and Danielle Costa Morais ${ }^{2 *}$
}

Received December 2, 2011 / Accepted August 20, 2013

\begin{abstract}
Any interaction involving individuals, whose objectives are conflicting with each other, may establish a negotiation process. In a negotiation, each party should develop his/her own strategy and, normally, a win-lose vision is frequently adopted. The main consequence of this behavior is a result, in which both parties lose, especially when the negotiation involves more than one aspect, such as negotiations resulting from purchases of material for construction industry, where aspects like price, quality and lead-time should be considered. Most of the negotiation involving construction industry adopts a win-lose vision; and, commonly, only the issue price is considered. The goal of this paper is to propose a framework to support negotiations between two parties (buyer and seller) in the supply chain of construction industry. The combination of a win-win strategy with a multicriteria analysis produces a best compromise solution for both parties. A simulation of negotiation using realistic data is presented.
\end{abstract}

Keywords: negotiation support system, multicriteria analysis, construction industry.

\section{INTRODUCTION}

A negotiation between buyers and suppliers arises from the existence of conflicting objectives among the parties in a purchase. For example, as far as objective price is concerned, suppliers always want the highest profit while buyers wants to pay as little as possible for a product or service that is being negotiated.

In the negotiation process, each party (buyer or seller) develops his own strategy in order to achieve his objective; also, usually a win-lose behavior is adopted. A possible consequence of this behavior is that, in some situations, both parties lose, especially when the negotiation process involves other issues beyond the price. For example, from the point of view of the buyer, he can achieve the lowest price in the purchase, however the acquired product or service has a very low quality; in this case, the seller also loses since a product/service with a very low quality can affect the trust between the parties.

\footnotetext{
*Corresponding author

${ }^{1}$ Federal University of Campina Grande, PB, Brazil. Academic Unit of Production Engineering.

E-mail: fernandoschramm@globo.com

${ }^{2}$ Federal University of Pernambuco, Production Engineering Department. E-mail: daniellemorais@yahoo.com.br.
} 
Therefore in order to improve the quality of a negotiation, a win-win behavior should be adopted instead of win-lose behavior. Consequently, the parties should accept trade-offs between different issues involved in a purchase. These trade-offs can be assessed through a structured negotiation process. Several methods were developed in order to support negotiation process: Lim \& Benbasat (1993), Holsapple et al. (1998), Sandholm et al. (1999), Sandholm (2000), Lomuscio et al. (2003), Klein et al. (2003), Luo et al. (2003), Lai et al. (2004), Lai et al. (2006), Guo \& Lim (2007), López-Carmona et al. (2009) and Zhang et al. (2011).

Some authors point out that the supplier selection is a decision-making problem with multiple issues (Weber et al. 1991; Holt, 1998; Degraeve et al., 2000; De Boer et al., 2001; Ha \& Krishnan, 2008; Wu et al., 2010; Ho et al., 2010). Dickson (1966) created a set with 23 issues, which he thinks should be considered in a supplier selection.

In this sense, a multi-criteria analysis can be an important tool to support decisions related to suppliers' selection, in which multiple criteria are being considered (Schramm \& Morais, 2012). This kind of analysis supports decision-makers in organizing and synthesizing such information in a way which leads them to feel comfortable and confident about making a decision, guaranteeing that all criteria involved in the decision process will be properly taken into account (Belton \& Stewart, 2002).

Some suppliers selection models discussed in the specialized literature adopt a multicriteria approach in their evaluation processes (Weber et al. 1991; Holt, 1998; Degraeve et al., 2000; De Boer et al., 2001; Ha \& Krishnan, 2008; Wu et al., 2010; Ho et al., 2010). However, these models are based on a singleton values for assessing the performance of the suppliers in the issues considered. This kind of evaluation is very restrictive for performing bargain between the parties in order to achieve better results for both in purchasing process. The use of an evaluation process based on interval values for the offers can increase the region of negotiation between the parties and, consequently, improve the final results for both buyer and supplier.

As far as construction industry is concerned, the supplier selections processes do not adopt a multicriteria approach - only the issue related to price of materials is considered. As a consequence, other important issues in a purchase negotiation process are neglected. This behavior was diagnosed in a survey conducted by Verma \& Pullman (1998), whose results indicated that, although managers of different supply chains said that the aspect quality is the most important objective to be considered in supplier evaluating, they select the suppliers according to the lowest price offered by them and their delivery performance. This limitation of the negotiation process undermines the achievement of other goals and, paradoxically, affects the profitability of the business.

The gaps identified in the supplier selection processes discussed in the specialized literature, particularly the models that are intended to the construction industry, motivated the development of a protocol for suppliers' selection in construction industry, which combines multicriteria analysis and negotiation techniques. The core of this protocol is a framework to support bilateral and multi-issue negotiations between construction companies (buyers) and suppliers (sellers). 
The remainder of this paper is organized as follows. After the background for negotiation in Section 2, we present the general protocol for suppliers' selection in Section 3. The proposed negotiation framework and its example are presented in Sections 4 and 5, respectively. Finally, in Section 6, the conclusions are drawn.

\section{BACKGROUND FOR NEGOTIATIONS}

According to Wang \& Zionts (2008), negotiation is a way for parties to reach an agreement in a dispute or in a joint decision-making. A negotiation is appropriated in situations in which the involved parties have different interests and values (Raiffa, 1982).

In human negotiations, the negotiators' bargaining determines the price or other issues. However, a negotiation related to multiple issues becomes more complex than one involving only one (Lai et al., 2006).

In this sense, it is possible to develop a classification for the negotiation problems according to the number of parties and issues involved. The typologies have own characteristics and they require the development of a specific theoretical framework which should aim to build a contention agreement. A negotiation among two parties or more can be classified as distributive or integrative (Raiffa, 1982).

\subsection{Distributive negotiation}

In distributive negotiations, only one issue is considered and the parties have almost strictly opposing interests on that issue. Whether a party gets more, less another party gain and they want as much as they can get with some exceptions. Of course, if a party is too greedy or if its opponent is too greedy, or if both are too greedy, they both will fail in the process to reach an agreement that would mean profits or incomes for both (Raiffa, 1982).

Wang \& Zionts (2008) simulated distributive negotiation in order to verify the agreement effectiveness involving one single issue (such as price). Integrative negotiations involve more than one issue; this kind of negotiation is more complex than negotiations involving only one (Lai $e t$ al., 2006). The following section presents some aspects of integrative negotiation.

\subsection{Integrative negotiation}

According to Zhang et al. (2011), in integrative negotiation, involving multiple issues, a decision of a party depends on her/his preference on all issues considered. A traditional way to make tractable integrative negotiation involves primarily characterizing the preferences with a utility function. In this sense, negotiators make decisions based on their respective utility functions for different aspects (issues). However, Lai et al. (2004, 2006) emphasize that it is not trivial to define each utility function for all issues related to the negotiation, especially when there are conflict in the preferences of multiple issues, i.e., how much an individual is willing to lose in one issue to win in another or vice versa. This will depend on the multi-issue utility function 
that an individual will get when all issues are being considered simultaneously in the negotiation process. Nevertheless, the preference elicitation process as recommended in the Multi-Attribute Utility Theory (MAUT) (Keeney \& Raiffa, 1976) requires much time and, in some cases, it is considered intractable.

Lai et al. (2004) argue that multi-issue negotiation is a useful mechanism due to its advantage to generate win-win solutions for both parties. A win-win result can be obtained through trade-offs among the possible gains that can be obtained in the different involved issues. In the searching for win-win solutions, Lai et al. (2004) argue that rational negotiators should not "leave extra money on the table". Another characteristics of integrative negotiations is that the parties are not strict competitors and it is no longer true that if one party gets more, the other party necessarily has to get less - both can be winners in this process. They can cooperate in order to increase the size of the pie that they eventually will have to divide (Raiffa, 1982).

As a consequence of the above characteristics, the concept of Pareto-optimal solution emerges: Pareto-optimal solution is a solution that cannot be improved further without sacrificing someone's utility, i.e., if there is another solution from which a party may get more than from this Pareto-optimal solution, then the other party must get less by that another solution.

Klein et al. (2003) proposed a mediation framework for negotiations involving complex contracts, where there are multiple parties negotiating about many aspects. According to Zhang et al. (2011), the proposed framework is a good example of an approach to solve the problem of interdependence between issues.

Lai et al. (2006) developed a Pareto-efficient model for negotiation problems with multi-issue with the following characteristics: parties are self-interested, information is incomplete, and the utility functions are nonlinear and not explicitly known. Their model includes an unbiased mediator, who applies a query learning to keep the agreements nearest from the set of Paretoefficient solutions, named Pareto-Efficient Frontier (PEF), without heavy computing. Lin \& Chou (2004) also proposed a mediation protocol that can be applied in a bilateral and multiple issues negotiation with the uncertainty for both parties, who are no self-interested.

According to Lai et al. (2004), before starting a multi-issue negotiation, the parties must decide about two aspects: (a) procedure and (b) implementation. These two decisions is the base for a framework for multi-issue negotiations (Fig. 1).

In relation to the procedure, the parties can choose one of the following options: separate negotiation, simultaneous negotiation and sequential negotiation. In the separate negotiation, the parties negotiate each issue separately (independently and simultaneously); the sequence of the issues is irrelevant. In the simultaneous negotiation, both parties negotiate a complete package related to all issues, simultaneously. In the sequential negotiation, both parties negotiate issue by issue sequentially, i.e., this is the issue-by-issue negotiation where the negotiators must also decide the order in which they will negotiate each issue; a negotiation agenda is necessary. 


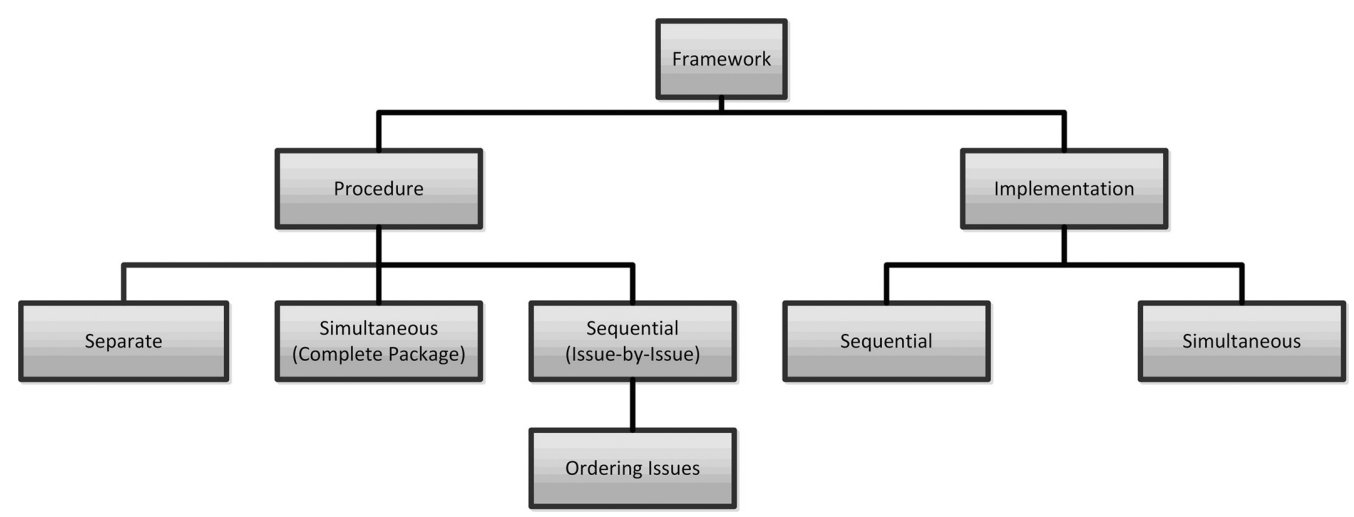

Figure 1 - Framework for the types of multi-issue negotiations (Source: Lai et al., 2004).

The agreement implementation can be sequential or simultaneous. In the sequential case, when an agreement in one of the issues is reached, then it is implemented; while, in the simultaneous case, the agreements are implemented jointly, after reaching a joint agreement about all issue.

Lai et al. (2004) performed a literature review in the multi-issue negotiation field and they defined that there are three types of research related to Game Theory and Artificial Intelligence (AI): (i) issue by issue negotiation, (ii) multi-issue cooperative negotiating, and (iii) multi-issue negotiation with heuristics methods. In the first type, an agreement is built through a multi-issue negotiation strategy, which examines each aspect individually and interactively; in addition, the frameworks consider parties as non-cooperative and they are built for environments with incomplete and asymmetric information, where an agenda containing the order in which issues will be treated is proposed. In the second type is used a multi-issue concession strategy based on additive aggregation model and the PEF (Nash, 1950; Keeney \& Raiffa, 1976; Raiffa, 1982), whose parties are considered cooperative and they have complete and symmetrical information about their environments. In the last type, an agreement is reached through a hybrid negotiation strategy, which uses the first two types of theoretical framework with the focus in automated models based on autonomous agents for multi-issue negotiation and in negotiation strategies tractable. The last type is related to AI. An autonomous agent is a computer system located in a particular type of environment, which is capable of autonomous action in order to achieve their projected goals. The agent-based technology has emerged as a new paradigm for conceptualizing, designing and implementing software systems (Jiao et al., 2006).

\subsection{Negotiation Support Systems (NSS)}

Guo \& Lim (2007) point out that NSS are a special support systems class developed to seek consensus and to solve conflicts in negotiation problems. They are designed to assist parties in reaching mutually satisfactory decisions through supporting information analysis and communication protocols. The works in this field are focused in the theoretical framework for design and implementation of NSS (Holsapple et al., 1998; Lim \& Benbasat, 1993; López-Carmona et al., 
2009), as well as in the negotiation problems related to modeling and representation (Sandholm et al., 1999; Sandholm, 2000; Lomuscio et al., 2003; Klein et al., 2003; Luo et al., 2003; Lai et al., 2004, 2006; Guo \& Lim, 2007; Zhang et al. 2011; etc.).

NSS are designed to aid negotiators during the different negotiation process stages and they are used to (Braun et al., 2006): (i) structure and analyze a particular negotiation problem; (ii) elicit preferences and use them to construct a utility function; (iii) determine feasible and efficient alternatives; (iv) define a set of tactics for negotiation; (v) visualize different aspects of the problem and the process; and (vi) facilitate communication.

There are fifteen postulates for a NSS design activity, which are distributed among the definitions of the eight basic parameters for the negotiation process. These parameters are in Holsapple et al. (1998) theoretical framework and will be described on Section 4.1. Guo \& Lim (2007) declared that any negotiation could not be understood without the definition of these eight parameters.

\section{GENERAL PROTOCOL FOR SUPPLIERS SELECTION}

The general protocol to achieve a deal defines the procedures that are necessary to execute the phases of the process for suppliers' selection: (i) invitation; (ii) bidding; and (iii) designation. The protocol will be used as basis for the establishment of the initial proposal of the negotiation process to be conducted between the construction company and its suppliers.

The invitation phase is divided into five steps: (a) definition of aspects; (b) formulation of invitation-letters; (c) sending invitation-letters to suppliers; (d) production of offers by suppliers; and (e) submission of offers by suppliers. In the first step, the manager responsible for purchase will define the issues, which must be considered during the suppliers selection of building materials; for example, lead time, price and quality. In the second step, he/she will formulate the invitation-letters and, in the third step, he/she will send these letters to the potential suppliers who comply with company requirements, inviting them to submit their offers. In the fourth step, the invited suppliers will evaluate the issues, which are being considered by the company; then, they will prepare their offers to be sent to the company. In the last step, the invited suppliers will submit their offers to the construction company. The Figure 2 shows the flowchart for the invitation phase.

The bidding phase is divided into three steps: (a) evaluation of proposals; (b) negotiation with suppliers; and (c) signing a contract. In the first step, the construction company will create an evaluation matrix according to the framework presented in Section 4. Then, during the second step, the company will conduct the negotiation process itself based on the framework. Finally, during the third step, an agreement over each issue negotiated is achieved and a contract between the company and the supplier, who had better performance, is signed. The Figure 3 shows the flowchart for the bidding phase, emphasizing the negotiation step, since it is the main goal of this paper.

During the designation phase, a list with building material and its respective suppliers will be created. Moreover, it will be created a schedule for delivery, which will be based on the production schedule of the construction company. The Figure 4 shows the flowchart for the designation phase. 


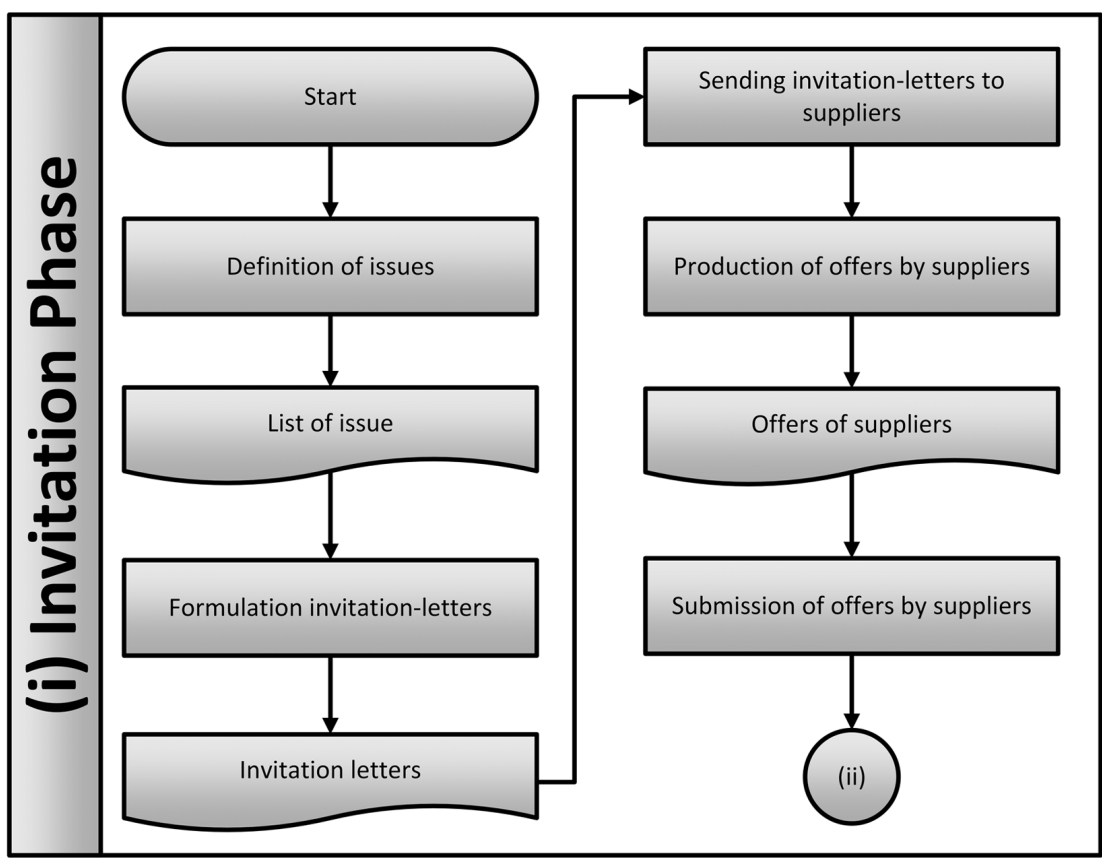

Figure 2 - Flowchart for the invitation phase.



Figure 3 - Flowchart for the bidding phase.

\section{PROPOSED FRAMEWORK}

In this section, we describe a bilateral and multi-issue NSS proposed framework based on mediation, which may be used in the negotiation step showed in the bidding phase of the general protocol for supplier selection by construction companies (buyers) and their suppliers (sellers). 


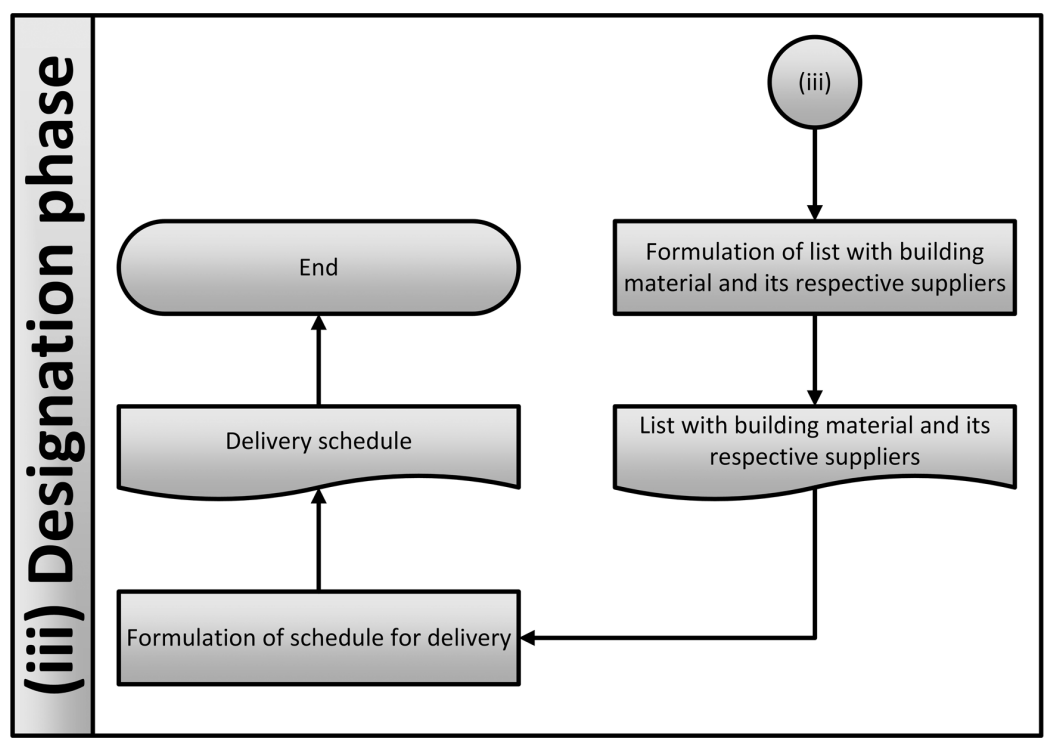

Figure 4 - Flowchart for the designation phase.

Chen \& Huang (2007) have already proposed a model for selection of suppliers, which combines multicriteria analysis and negotiation approaches. The core idea of that model is a selection process, supported by a multicriteria method, followed by a negotiation with the selected candidate only. Here, we intend to create a mechanism to support negotiation with all suppliers, who submitted proposals according to a protocol, and the selection process can be performed based on the suppliers' performance during the negotiation.

According to the Figure 1, the type of negotiation procedure adopted in this proposed framework is simultaneous and the type of agreement implementation is also simultaneous.

Negotiations that took place at the bidding phase has the following features and rules: two parties bargaining - construction company and suppliers; parties are monolithic; negotiation can be repetitive, however, there is not linkage effects (for example, if a supplier is able to sell two materials for the construction company, the effects of one negotiation will not affect other negotiations); there are more than one issue to be negotiated; an agreement is necessary, i.e., the construction company will reach an agreement with one supplier of each material; ratifications are not needed; threats are not possible; there are restrictions of time and costs related to time, even assuming that the negotiation will take place in advance before the start of construction; a contract is mandatory; the negotiations are private, i.e., the parties will not disclose negotiations details with the population which will be affected by the negotiation process; both parties are considered fully cooperative partners; there is a mediator in the negotiation process.

Based on those features and rules of negotiation, the framework to support the negotiation process will be developed below. It will take into account the issues defined in the invitation phase and the offers submitted by suppliers in that phase. The flowchart with the proposed NSS procedures is shown in Figure 5. 


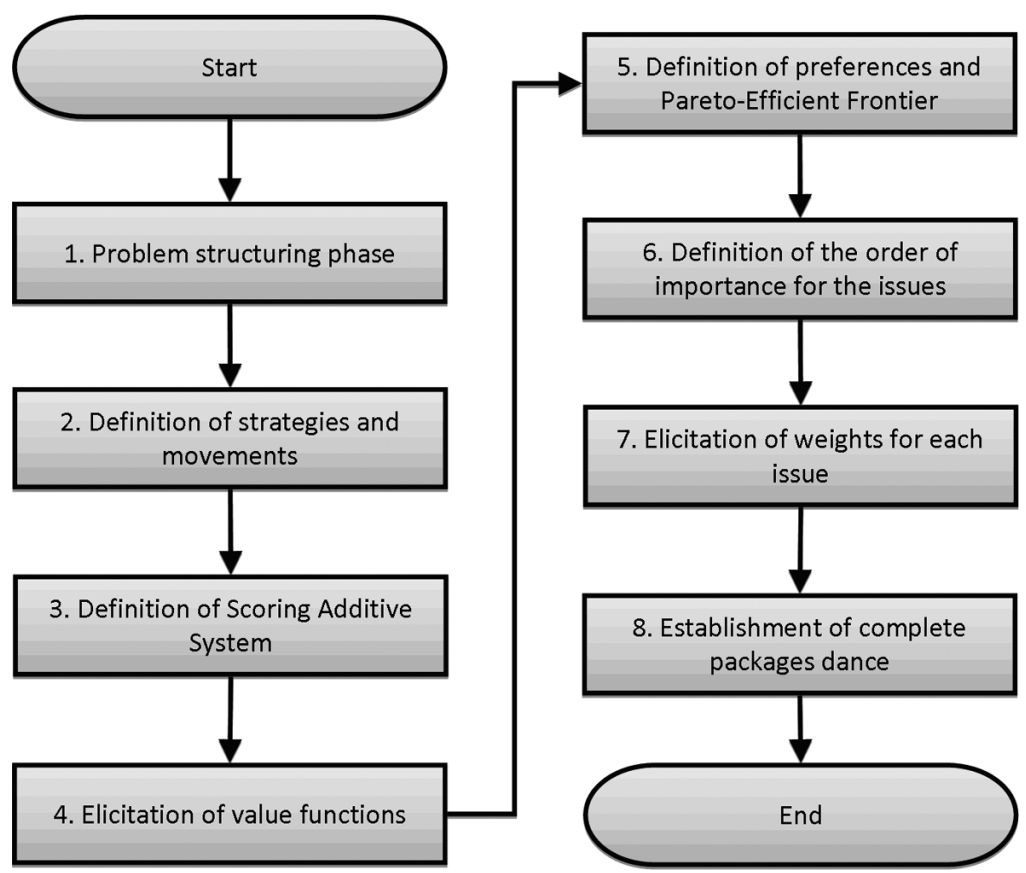

Figure 5 - Flowchart of NSS proposed.

\subsection{Problem structuring phase}

The NSS is structured according to the Holsapple et al. (1998) theoretical framework:

$$
N=(I, E, A C C E P T, L O C, S, M, R, A),
$$

where, $I$ represents the issues and their respective spaces, $E$ denotes the set of the entities involved, ACCEPT indicates the agreement region of parties in the issues space and LOC represents the parties location in the negotiation process, i.e., where the parties' offers performance are located in the issues space at instant time $t . S$ and $M$ are the strategies and the movements of entities, respectively. $R$ indicates the negotiation rules, which have been defined in the beginning of the proposed framework, and $A$ denotes assistance and support by intervenors. In this work, the mediator is the type of intervenor used to support the parties in the negotiation.

The mediator is an impartial observer who tries to support negotiators in their investigations to find an agreement. He/she supports the negotiation process, but he/she does not have any authority to dictate a solution. Several negotiation frameworks based on this mediation concept are proposed in the literature. According to Zhang et al. (2011), the first framework was proposed by Fisher (1978), which was improved by Ehtamo \& Hämäläinen (2001). Lin \& Chou (2004) also proposed a mediation framework based on the approach suggested by Fisher (1978). All methods described by these authors are based on cooperative negotiators who cannot be regarded as self-interested in negotiation process. 
In integrative negotiations, parties are often faced with hard decisions, which cannot be made through a rational strategy. A barrier may arise, if both negotiators refuse to make concessions, simultaneously. Because of the fear in losing the offer advantages for the opponent, seller and buyer are not willing to be the first to give an offer. Mediation is required in those situations (Zhang et al., 2011).

To establish the variables $I$ and $E$, the mediator will carry out the development of a decision matrix associating the set of alternatives (suppliers) with the criteria (issues), where rows represent suppliers and columns denote issues. The matrix size is $m \times n$, where $m$ is the number of suppliers and $n$ is the number of issues. Let $A_{i j}=\left(a_{i 1}, a_{i 2}, \ldots, a_{i n}\right)$ as the supplier $i^{\text {th }}$, where $a_{i j}$ is the one-dimensional performance of supplier $i$ in the issue $j(j=1,2, \ldots, n)$. The invitation phase will be used as input in this procedure.

Then, to establish the variable $A C C E P T$, the mediator together with the parties will define the best and worst agreements which are related to the best and worst expected performances in the offers, according to construction company and supplier point-of-view. The variable $L O C$ at instant time $t=0$ will be related to the offers made in the invitation phase and at instant $t=1$ will be related to Pareto-efficient solution.

\subsection{Definition of strategies and movements for the negotiation}

In a negotiation, the strategies and movements are developed in order to maximize the parties' gains. Each party may develop specific movements and strategies, which will be used with the utility or value functions definition for different issues. The strategies and movements are represented by the $S$ and $M$ parameters of the Holsapple et al. (1998) NSS theoretical framework.

During this procedure implementation, parties may consider the following questions (Holsapple et al., 1998): Should I change my acceptance region? Should I attack all dimensions of an issue as a whole package or settle them one by one? If handled one by one, what should the sequence be? Should I solve the easy one first or the difficult one first? On which dimensions should I remain firm and on which should I be more flexible? Could I use some linkage strategy among the dimensions of an issue? What kind of pace should I have in making concessions? How open should I be? Should my initial location be reasonable or should it be extreme, allowing me greater latitude for adjustments? For the authors, the answers will shape party's strategy and this strategy plays a crucial role, making movements in the issue solution space.

According to Holsapple et al. (1998), for each party $e_{i}$ (where, $i=1,2, \ldots, I$ ) there should be a strategic function $(S)$ as the following:

$$
S_{i}^{t+1}=\Theta_{i}\left(I, L O C^{t}, e_{i}, R, A, t\right),
$$

where, $S_{i}^{t+1}$ is the set of candidate strategies for party $e_{i}$ which will be selected for pursuing a new location at time $t+1 ; \Theta_{i}$ is entity $e_{i}$ 's strategy function; $L O C^{t}$ is the set of all entities' locations at time $t$ (i.e., the current state of negotiation process). 
There are many negotiation strategies available in the literature (Zhang et al., 2011; Louta et al., 2008; Lai et al., 2006; etc.). In this paper, we adopt a concession strategy to determine the value levels desired by negotiators, it is based on Scoring Additive System (SAS) and on a simulation of the whole packages of offerings, named Complete Packages Dance (CPD). In order to find multi-issue Pareto-efficient outcomes we will also use a search strategy in PEF. The combined use of those strategies achieves maximum win-win outcomes for both parties. In addition, the proposed framework in this paper is focused in a bilateral and multi-issue negotiation, whose negotiators are considered cooperative and the information is symmetric. As they are fully cooperative negotiators, thus, there is only a single strategy for both parties.

In integrative negotiations, when is adopted a SAS the reserve prices of parties become the best compromise values reached for both, based on the CPD and the PEF.

\subsection{Definition of Scoring Additive System}

Raiffa (1982) define a SAS as a scoring system, in which seller and buyer assign values to different levels within each issue. This valuation will be performed through a tradeoff analysis between different issues. One way to valuate issue is assigning a physical performance (money, for example) to each of issues, but this alternative is not always convenient and interesting. In many situations, it is appropriate to develop a scoring system based on abstract linear value functions for seller and buyer, for example. If there are more than two issues and if the tradeoffs between levels within any two issues are preferentially independent in other issues, then, a SAS is appropriate for the negotiation problem. Moreover, it is important to notice that some negotiators begin the negotiation offering a fully package of offers, of course favorable to his/her side, and the opponent responds with another package of counter-offer. Then, it can be seen that the CPD corresponds to a set of all possible offers combinations, which both parties can offer during the negotiation. In our framework, the initial proposal will be formulated as established in the general protocol selection.

In the SAS proposed we suppose that (based on Raiffa, 1982): $i(i=1,2)$ denotes a negotiator; $j(j=1,2, \ldots, J)$ denotes an issue; $x_{j}$ is a general performance of $j^{\text {th }}$ issue with domain $\left[a_{j}, b_{j}\right]$ for $j(j=1,2, \ldots, J) ; V_{i j}\left(x_{j}\right)$ is the value related to negotiator $i$ on $j$ issue at $x_{j} ; w_{i j}$ is the importance weight assigned by negotiator $i$ on $j$ issue, where $\sum_{j=1}^{J} w_{i j}=1$ (for $i=1,2$ ); the total value for contract $x=\left(x_{1}, \ldots, x_{j}\right)$ is given by equation:

$$
V_{i}(x)=\sum_{j=1}^{J} w_{i j} V_{i j}\left(x_{j}\right)
$$

Moreover, if weight $V_{1}$ is 1 and $V_{2}$ is $\lambda$ (which can be varied to get different points on the efficient frontier) it is possible to see that

$$
V_{1}(x)+\lambda V_{2}(x)=\sum_{j=1}^{J}\left[w_{1 j} V_{1 j}\left(x_{j}\right)+\lambda w_{2 j} V_{2 j}\left(x_{j}\right)\right]
$$


Hence, if it is needed to choose $x$ to maximize $V_{1}(x)+\lambda V_{2}(x)$, then it is possible to choose $x_{j}$ in $\left[a_{j}, b_{j}\right]$ to maximize $w_{1 j} V_{1 j}\left(x_{j}\right)+\lambda w_{2 j} V_{2 j}\left(x_{j}\right)$, for $j=1, \ldots, J$. Let $x_{j}^{(\lambda)}$ be an optimum point for that $\lambda$ and let $x^{(\lambda)}=\left(x_{1}^{(\lambda)}, \ldots, x_{j}^{(\lambda)}, \ldots, x_{J}^{(\lambda)}\right)$. The joint evaluation $\left[V_{1}\left(x^{(\lambda)}, V_{2}\left(x^{(\lambda)}\right]\right.\right.$ will be a point on efficient frontier whose supporting tangent line is the locus of points for which $V_{1}+$ $\lambda V_{2}=V_{1}\left(x^{(\lambda)}\right)+\lambda V_{2}\left(x^{(\lambda)}\right)$. The analysis can be easily generalized to situations involving more than two negotiators, assuming $i=1,2, \ldots, I$ and in lieu of $V_{1}(x)+\lambda V_{2}(x)$ using $\sum_{i=1}^{I} \lambda_{i} V_{i}(x)$ for a set of $\left(\lambda_{1}, \ldots, \lambda_{I}\right)$ weights. In this case, $x_{j}^{(\lambda)}$ is a maximizer of:

$$
\sum_{i} \lambda_{i} w_{i j} V_{i}\left(x_{j}\right), \text { and all goes through as before. }
$$

Since the formalization of the SAS the mediator should clarify four factors: the type of value function to be adopted in every aspect; the negotiators preference on the offers package in the PEF; the method in which negotiators will establish the relative importance of issues; and how the weights values will be assigned to each issue.

In this sense, the proposed framework also contributes with NSS design and implementation processes through a literature research about theoretical approaches which can develop knowledge about those four factor implicitly addressed by Raiffa (1982). Those four factors are discussed below in order that they are listed above.

\subsection{Elicitation of value functions}

The mediator should elicit the value functions for each one-dimensional issue of negotiation. If uncertainty is considered, then this function should be elicited as established in one-dimensional utility theory and MAUT (Vincke, 1992; Keeney \& Raiffa, 1976).

The proposed framework does not consider uncertainty and it assumes a linear value function for each one-dimensional issue. The types of linear value functions are based on the Simple MultiAttribute Rating Technique (SMART) family of multi-criteria decision-aid methods, which are proposed by Edwards (1977) and Edwards \& Barron (1994).

This family uses the strategy of heroic approximation in order to justify the linear approximation of a one-dimensional value and the use of an additive aggregation model; the method also requires an additive independence between one-dimensional values of all criteria.

One of the advantages of the SMART family methods over other unique synthesis criterion methods, such as MAUT, is related to the fact that the decision makers find it easy to understand the concepts and parameters inherent in the method, which makes the preference modeling simpler and, consequently, increases the effectiveness of applying the methods. All these aspects make SMART based methods very appropriate to be applied on this proposed negotiation framework.

Therefore, besides to provide some standard shapes to the value functions, this family of methods also assists in the development of a procedure to determine the order of importance of the issues and their respective weights. Compensatory multi-criteria methods, such as SMART methods, 
have constant of scale, $w_{i j}$, that represents trade-offs among criteria; however, in the literature about SMART methods, the term weight is used instead of constant of scale.

\subsection{Definition of preferences and Pareto-Efficient Frontier}

PEF is a region in which there are possible maximum joint gains (Raiffa, 1982). In order to determine the efficient-frontier, it is necessary to collected information from both parties; thus, the parties must work in collaboration with each other. In our framework, the mediator will perform an individual analysis with the negotiator in order to identify the individual preferences for the packages through the assumptions presented in Nash (1950). Through this analysis, the mediator will perform the procedure to consolidate the CPD.

Negotiators, considered rational, can achieve an agreement about a PEF through Nash's axioms (Nash, 1950). These axioms were integrated to the Utility Theory (Keeney \& Raiffa, 1976; Raiffa, 1982) in order to allow that the preference of each individual, about the maximization of his/her gains in a negotiation, can be expresses by a mathematical model and, consequently, to achieve an agreement, which provides maximum joint gains.

In these axioms, the parties, involved in a negotiation, are considered completely rational. In this sense, they can compare, with precision, their preferences in relation to several aspects; they have the same skill to negotiate; and they have total information about the preference of his/her respective partner in negotiation process.

Based on these premises, it was formulated a general model to supports a negotiation, which attempts to these axioms. Suppose $A$ and $B$ are elements that compose a set $S$ of offers involved in a negotiation; suppose also that each party has his/her own preference for each offer. Let $p$ be the preference of a negotiator in relation to an offer $A$, where $0 \leq p \leq 1$, the preference of the same negotiator in relation to the offer $B$ is given by $(1-p)$. With the following assumptions, it is possible to apply the Utility Theory for a single individual:

1. A negotiator suggests two possible offers and he/she can decide which is preferable or if they are equally desirable.

2. The ordering produced in the first assumption is transitive; if $A$ is better than $B$ and $B$ is better than $C$ then $A$ is better than $C$.

3. Any probability combination of equally desirable states is just as desirable as either.

4. If $A, B$, and $C$ are as in assumption 2 , then there is a probability combination of $A$ and $C$ which is just as desirable as $C$. This amount is related to an assumption of continuity.

5. If $0 \leq p \leq 1$ and $A$ and $B$ are equally desirable, then $p A+(1-p) C$ and $p B+(1-p) C$ are equally desirable. Also, if $A$ and $B$ are equally desirable, $A$ may be substituted for $B$ in any desirability ordering relationship satisfied by $B$. 
The resulting utility function of the Nash (1950) axioms is not unique, i.e., if $u$ is such function, then it is also a linear equation given by $a u+b$, where $a>0$. Moreover, such utility function must satisfy the following assumptions: (a) $u(A)>u(B)$ is equivalent to $A$ is more desirable than $B$, etc.; (b) if $0 \leq p \leq 1$, thus, $u[p A+(1-p) B]=p u(A)+(1-p) u(B)$.

Until this moment we have addressed the axioms for each single negotiator; however, as in a negotiation will be involved at least two negotiators we must enlarge the assumptions for these situations. In general, Nash (1950) defines the joint probability of any two negotiators as a combination of their individual probabilities. Thus, if $\left[x_{1}, x_{2}\right]$ is the joint probability of two parties for $A$ offer, if $\left[y_{1}, y_{2}\right]$ is the joint probability to $B$ offer and if $0 \leq p \leq 1$, thus $p\left[x_{1}, x_{2}\right]+(1-p)\left[y_{1}, y_{2}\right]$ will be defined as $\left[p x_{1}+(1-p) y_{1}, p x_{2}+(1-p) y_{2}\right]$. Give that $u_{1}$ and $u_{2}$ are the utility functions for two negotiators and given that $c(S)$ represents the solution point (or the agreement point between the parties) in a set $S$ which is convex and compact and it includes the origin, so we assume that:

6. If $\alpha$ is a point in $S$ such that there exists another point $\beta$ in $S$ with the property $u_{1}(\beta)>$ $u_{1}(\alpha)$ and $u_{2}(\beta)>u_{2}(\alpha)$, thus $a \neq c(S)$. This assumption shows the idea of each negotiator wants maximize his/her own utility in final agreement of bargaining process.

7. If the set $T$ includes the set $S$ and $c(T)$ is in $S$, thus $c(T)=c(S)$. It is said that a set $S$ is symmetric if there are utility operators $u_{1}$ and $u_{2}$ such that when $(a, b)$ is contained in $S$, $(b, a)$ is also contained in $S$, such that the graph becomes symmetrical with respect to the line $u_{1}=u_{2}$. This assumption shows the following idea: If two rational individuals would agree that $c(T)$ would be a fair bargain and if $T$ were the set of possible bargains, then they should be willing to make an agreement, lesser restrictiveness. It is not to attempt to arrive at any bargains represented by points outside of the set $S$, if $S$ contained the point $c(T)$. If $S$ were contained in $T$ this would reduce their situation to one with $S$ as the set of possibilities. Hence, $c(S)$ should equal $c(T)$.

8. If $S$ is symmetric and $u_{1}$ and $u_{2}$ show this, thus $c(S)$ is a point in the form $(a, a)$, i.e., a point on the line $u_{1}=u_{2}$. This assumption shows the equality of bargaining skill related to negotiators in bargaining process.

These assumptions define that the Pareto-efficient solution is a point in the set $S$, which is located in the first quadrant of the corresponding graph, where the two negotiators utilities will be maximized. This point exists because of the compact size of this set. In addition, the convexity property of this set makes this point of agreement unique.

\subsection{Definition of the order of importance for the issues}

The order of relative importance for the issues will be established through step 7 of the SMARTS (Simple Multi-Attribute Rating Technique, Swing Weights) method proposed by Edwards (1977) and Edwards \& Barron (1994) and it should be done for both company and suppliers. As far as 
SMARTS method is concerned, the seventh step in this method yields the rank order of the issues, i.e. the mediator will ask negotiators the questions proposed in the method, whose goal will be build a rank order of the issues.

\subsection{Elicitation of weights for each issue}

The SMARTER (Simple Multi-Attribute Rating Technique, Exploiting Ranks) method will be used in this procedure. It is divided into nine steps, which includes the first six steps of SMART method, the seventh step of SMARTS method, which is the swing weights, correcting the intellectual error of SMART when defining a ranking of criteria according to their relative importance (Edwards \& Barron, 1994); the eighth step of SMARTER which establishes the weight value of a criterion according to its respective ranking based on the Rank Order Centroid (ROC) approach; and finally the nineth step is to decide (Edwards \& Barron, 1994 and Barron \& Barrett, 1996).

Barron \& Barrett (1996) developed a formally justified approach whose purpose was to create a procedure for values' elicitation related to the weights of each issue. This approach establishes the weights of issues according to their relative importance, which has been defined in the procedure 6 of our framework proposed. This approach is called Rank Order Centroid (ROC) or simply ROC weights. Therefore, the mediator will use this approach to define the values of the weights for every issue, according to builder and supplier point-of-view.

As far as SMARTER method is concerned, the assessment of criteria weights values in this method is simpler than other SMART family methods, since it uses the ROC approach to calculate the weights. ROC weights lead to the identification of the best compromise option 75 to $87 \%$ of the time, depending on simulation details. In the ROC approach proposed by Barron \& Barrett (1996) the weights are calculated according to the following expression:

If $w_{1} \geq w_{2} \geq \cdots \geq w_{k}$ then,

$$
\begin{aligned}
w_{1} & =(1+1 / 2+1 / 3+\cdots+1 / K) / K \\
w_{2} & =(0+1 / 2+1 / 3+\cdots+1 / K) / K \\
w_{3} & =(0+0+1 / 3+\cdots+1 / K) / K \\
w_{K} & =(0+\cdots+0+1 / K) / K
\end{aligned}
$$

Usually, if $K$ is the number of issues, then the weight of the $k^{\text {th }}$ issue is:

$$
w_{k}=(1 / K) \sum_{i=k}^{K}(1 / i) \text {. }
$$

\subsection{Establishment of Complete Packages Dance and outcomes analyses}

In this procedure will be established the fully packages of offers for both builder and for suppliers. This procedure is performed through a simulation of CPD. The mediator will analyze the simulation with the purpose to present the "fully package solution" which will be considered the 
best compromise solution. This "package" is the best compromise solution for both, that is, it is the solution found in PEF and it satisfies the Nash axioms described above. This solution if can be better for any of the negotiators, it will give worse value for another.

\section{EXAMPLE OF APPLICATION}

This example is based on a selection of suppliers to multi-use mortar, used by a construction industry in Recife, Pernambuco, which was supported by a multicriteria model proposed by Schramm \& Morais (2012). The following issues were considered in the negotiation of multiuse mortar: lead time (I1), transports costs (I2), failure rate (I3) and price (I4).

In relation to the strategic behavior adopted by the parties, it was assumed that they are fully cooperative negotiators, thus, there is only a single strategy for both parties, $\Theta_{\text {buyer }}=\Theta_{\text {seller }}$, which is represented by the function $\operatorname{Max}_{b}\left(P^{k}\right)+V_{s}\left(P^{k}\right)$, where $V_{b}$ and $V_{s}$ are the values functions assigned to the buyer and seller, respectively, and $P^{k}$ is an $k^{\text {th }}$ point of the region of acceptable offers. The region of acceptable offers is defined according to the information presented by the parties related with the best and worst agreement for each issue, according to the point of view of each party.

During the selection process performed by Schramm \& Morais (2012), the company received five offers from its suppliers. A negotiation, supported by the framework, between the company and the fourth supplier $\left(A_{4}\right)$ will be simulated, in order to show how the proposal works. The set of offers presented by $A_{4}$ during the ending of invitation phase (production and submission of offers by suppliers) is: $\mathrm{I} 1=10$ days; $\mathrm{I} 2=\mathrm{R} \$ 1.43 ; \mathrm{I} 3=7.93 \%$; and $\mathrm{I} 4=\mathrm{R} \$ 7.19$.

Considering a negotiation process with the supplier $A_{4}$, the best and worst agreement for both parties were defined according to the rule established in the problem structuring phase (Table 1). The best performance complete package of the buyer $\left(\alpha^{+}\right)$and the best performance complete package of the seller $A_{4}\left(\beta^{+}\right)$are assumed to have the highest value (100). On the other hand, the worst performance complete package of the buyer and of the seller $A_{4}, \alpha$ and $\beta_{-}$, respectively, are assumed to have the lowest value (0).

Table 1 - Best and worst agreements.

\begin{tabular}{|l|c|c|c|c|}
\hline \multirow{2}{*}{\multicolumn{2}{c|}{ Agreements }} & \multicolumn{4}{|c|}{ One-dimensional performance } \\
& \multicolumn{4}{|c|}{ for each Issue } \\
\cline { 2 - 5 } & $\mathrm{I} 1$ & $\mathrm{I} 2$ & $\mathrm{I} 3$ & $\mathrm{I} 4$ \\
& days & $\mathrm{R} \$$ & $\%$ & $\mathrm{R} \$$ \\
\hline Best Agreement for the buyer $\left(\alpha^{+}\right)$ & 5 & 1.10 & 7.60 & 7.19 \\
Worst Agreement for the buyer $\left(\alpha_{-}\right)$ & 18 & 1.85 & 12.30 & 10.95 \\
Best Agreement for the seller $\left(\beta^{+}\right)$ & 18 & 1.85 & 12.30 & 10.95 \\
Worst Agreement for the seller $\left(\beta_{-}\right)$ & 5 & 1.10 & 7.60 & 7.19 \\
\hline
\end{tabular}

The procedure for elicitation of value function allows the mediator to define the types of value functions for each aspect and each party. For the company (buyer), all issues are assumed to have 
the function type $b$ of SMART method, while for the supplier (seller), all issues are assumed to have the function type $a$ of SMART method (Edwards \& Barron, 1994). Figure 6 illustrates two graphics, which describes the linear one-dimensional value functions for issue I3 and for issue I4, according to the point of view of the company and the seller, respectively.
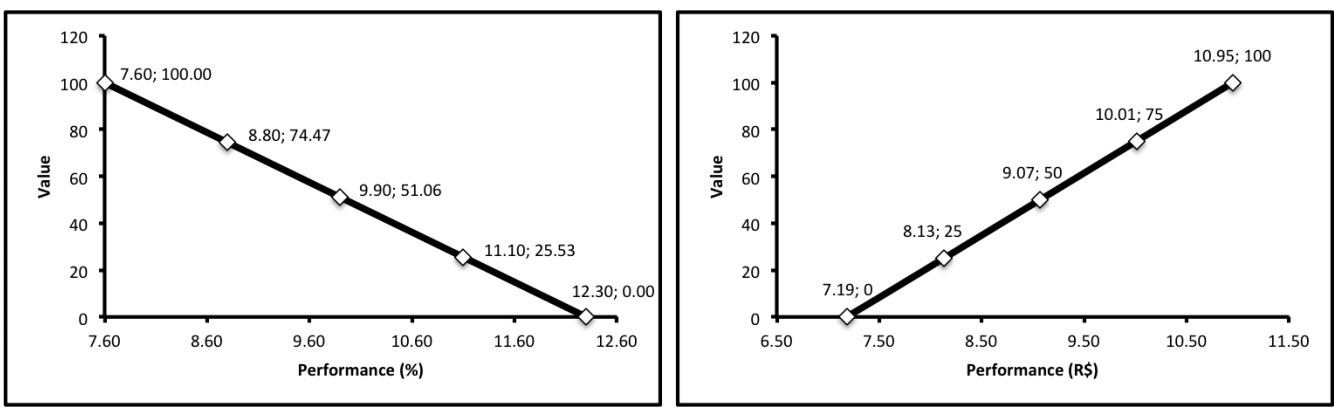

Figure 6 - (a) Company's value function for I3 and (b) Supplier's value function for I4.

The preference of the parties for the offer packages are based on the assumptions assumed during the procedure to define preferences. Then, it was performed the procedure to define the order of importance among the issues, presented in Table 2.

Table 2 - Ranking of importance among the issues.

\begin{tabular}{|c|c|c|}
\hline Issues & $\begin{array}{c}\text { Ranking of importance } \\
\text { (Company) }\end{array}$ & $\begin{array}{c}\text { Ranking of importance } \\
\text { (Supplier) }\end{array}$ \\
\hline I1 & $4^{\circ}$ & $2^{\circ}$ \\
I2 & $3^{\circ}$ & $3^{\circ}$ \\
I3 & $1^{\circ}$ & $4^{\circ}$ \\
I4 & $2^{\circ}$ & $1^{\circ}$ \\
\hline
\end{tabular}

According to the rules established on the proposed framework, the elicitation of weights of issues will be based on the method proposed by Barron \& Barrett (1996). The Table 3 shows the weights for each issue.

Table 3 - ROC weights for issues.

\begin{tabular}{|c|c|c|c|c|}
\hline \multirow{2}{*}{ Weights } & \multicolumn{4}{|c|}{ Issues } \\
\cline { 2 - 5 } & $\mathrm{I} 1$ & $\mathrm{I} 2$ & $\mathrm{I} 3$ & $\mathrm{I} 4$ \\
\hline Company & 0.0626 & 0.1458 & 0.5208 & 0.2708 \\
Supplier & 0.2708 & 0.1458 & 0.0626 & 0.5208 \\
\hline
\end{tabular}

Finally, a CPD for each 625 packages was established. To determine the better packages, it was calculated the total value, which corresponds to the sum of company's value $V_{b}\left(P^{k}\right)$ and supplier's value $V_{s}\left(P^{k}\right)$ for each package, then, the packages were ranked in crescent ordering of their total value. 
The Table 4 presents a sample of 15 fully packages and its respective values. The package $P^{625}$ corresponds to the best compromise agreement and the package $P^{1}$ corresponds to the worst agreement. The others packages are the ones which have the 13 betters results into the 625 available results.

Table 4 - Sample of fully packages.

\begin{tabular}{|c|c|c|c|c|}
\hline Packages & \multicolumn{4}{|c|}{ Values } \\
\hline$k$ & $P^{k}$ & $V_{b}\left(P^{k}\right)$ & $V_{s}\left(P^{k}\right)$ & $V_{b}\left(P^{k}\right)+V_{s}\left(P^{k}\right)$ \\
\hline 506 & $18 ; 1.66 ; 07.6 ; 10.95$ & 55.77 & 90.05 & 145.82 \\
501 & $18 ; 1.85 ; 07.6 ; 10.95$ & 52.08 & 93.74 & 145.82 \\
$\mathbf{5 2 1}$ & $\mathbf{1 8 ; 1 . 1 0 ; 0 7 . 6 ; 1 0 . 9 5}$ & $\mathbf{6 6 . 6 6}$ & $\mathbf{7 9 . 1 6}$ & $\mathbf{1 4 5 . 8 2}$ \\
516 & $18 ; 1.29 ; 07.6 ; 10.95$ & 62.97 & 82.85 & 145.82 \\
511 & $18 ; 1.47 ; 07.6 ; 10.95$ & 59.47 & 86.35 & 145.82 \\
522 & $15 ; 1.10 ; 07.6 ; 10.95$ & 68.10 & 72.91 & 141.02 \\
531 & $18 ; 1.66 ; 07.6 ; 10.01$ & 62.54 & 77.03 & 139.57 \\
537 & $15 ; 1.47 ; 07.6 ; 10.01$ & 67.68 & 67.08 & 134.77 \\
551 & $18 ; 1.85 ; 07.6 ; 09.07$ & 65.62 & 67.70 & 133.32 \\
514 & $08 ; 1.47 ; 07.6 ; 10.95$ & 64.28 & 65.52 & 129.80 \\
447 & $15 ; 1.10 ; 08.8 ; 09.07$ & 68.35 & 48.47 & 116.82 \\
424 & $08 ; 1.10 ; 08.8 ; 10.01$ & 64.95 & 46.91 & 111.86 \\
1 & $18 ; 1.85 ; 12.3 ; 10.95$ & 0.00 & 100.00 & 100.00 \\
625 & $05 ; 1.10 ; 07.6 ; 07.19$ & 100.00 & 0.00 & 100.00 \\
223 & $11 ; 1.10 ; 11.1 ; 08.13$ & 51.56 & 30.18 & 81.74 \\
\hline
\end{tabular}

Observing the packages $P^{506}, P^{501}, P^{521}, P^{516}$ and $P^{511}$, we note that they have a total value equal to 145.82 ; however, only the package $P^{521}$ complies with the assumptions assumed in the procedure for preference definition and for PEF definition. In the other packages, if the supplier's value increases, then the company's value decreases and vice-versa. Therefore, the package $(\mathrm{I} 1=18$ days; $\mathrm{I} 2=\mathrm{R} \$ 1.10 ; \mathrm{I} 3=7.60 \%$; $\mathrm{I} 4=\mathrm{R} \$ 10.95)$, with $V_{b}\left(P^{521}\right)=66.66$ and $V_{s}\left(P^{521}\right)=79.16$ may be chosen. At this moment, the parties could sign the contract required and conclude the bidding phase.

It is also noted that the package $P^{522}$ offers a bigger value to the Company than the package $P^{521}$ and, consequently, the purchase manager can suggest an agreement about this condition. To do this, the supplier must accept a reduction of about 6 points in his/her value.

The combined use of concession and search strategies on Pareto-efficient frontier allows achieve maximum win-win results for both parties. This solution would not have been easily found if the two parties were working alone in the negotiation process, that is, if they were not acting cooperatively. 


\section{CONCLUSIONS}

The paper presents a multicriteria additive framework to support a bilateral and multi-issue negotiation between a buyer and a material supplier (seller), in which the stakeholders can reach a deal based on their strategies of concession and search in the efficient Pareto-frontier. This system plays the role of a mediator, that is, it supports the negotiators to reach the Pareto-efficient optimal solution, which meets their own interests; however, the negotiators are responsible to decide if the solution, provided by the system, will be chosen to achieve the agreement. With this automated algorithm for negotiation, the negotiators could reach a Pareto-efficient contract with gains for both parties. Moreover, the mechanism for mediation is stable in the context of a negotiation, that is, the negotiators' behavior can be foreseen.

An important contribution is related with the development of strategies for negotiations between two parties, which allow that both reach multi-issues Pareto-efficient results. The combined use of concession strategies and investigation of efficient Pareto-frontier allows achieve maximum results for both parties. However, when negotiators do not accept the solution found by the proposed framework, thus a conflict situation may arise. In such situations, it will be necessary to develop a mediation protocol for solving conflicts.

The limitations of the traditional supplier selection models motivated the design of the new framework proposed in this paper. The overall supplier evaluation in the traditional models is performed on a singleton values for the offers in each issue, which limits the parties bargaining power as well as the set of possible solutions in each issue. In the proposed framework, an approach is structured in order to carry out bilateral negotiations. This approach allows an overall supplier evaluation based on interval values for the offers in each issue. This feature of the proposed framework increases the supplier bargaining power as well as his/her region of negotiation. The result of the framework can be used to construct a ranking of suppliers based on their overall performance on the negotiation process. Besides choosing the best supplier, the new proposal increases the construction company bargaining power that is prioritized in the proposed framework.

The development of the framework was intended to the construction industry due to the increasing of this industry in Brazil, since the last ten years. Consequently, the management problems which already existed in this supply chain became even more evident. For instance, in this supply chain the material price is commonly the only issue taken into account in the purchasing process; other important issues are neglected. In this context, the proposed protocol with a negotiation framework based on multiple issues is able to define structured procedures that improve the quality of the purchasing process and, consequently, the competitiveness of the construction industry supply chain.

In this paper we had focused in the proposal of a new framework for a negotiation process, showing an example of application based on a selection of suppliers, which was supported by a multicriteria model proposed by Schramm \& Morais (2012). Therefore, a real case study with the intention to analyze the feelings of the decision makers and how they feel comfortable can be focus of a future work. 
The application of the framework shows that negotiators do not need to provide key-information during the process. For future works, it is suggested the development of a protocol for communication between the parties involved, which is based on a mediated interventions, in order to attempt to the cases in which revelation of key-information is necessary.

\section{ACKNOWLEDGMENTS}

This work is part of a research program funded by the Brazilian Research Council (CNPq).

\section{REFERENCES}

[1] BARRON FH \& BARRETT BE. 1996. Decision quality using ranked and partially ranked attribute weights. Management Science, 42(11): 1515-1523.

[2] Belton V \& Stewart TJ. 2002. Multiple criteria decision analysis: An integrated approach. Kluwer Academic Publishers, Boston.

[3] Braun P, Brzostowski J, Kersten G, Kim JB, Kowalczyk R, Strecker S \& Vahidov R. 2006. E-Negotiation Systems and Software Agents: Methods, Models, and Applications. In: Intelligent Decision-Making Support Systems: Foundation, Applications and Challenges - Decision Engineering Series [edited by Gupta JND, Forgionne GA \& Mora M], Springer.

[4] Chen YM \& HUAng PN. 2007. Bi-negotiation integrated AHP in suppliers selection. Benchmarking: An International Journal, 14(5): 575-593.

[5] De Boer L, Labro E \& Morlacchi P. 2001. A review of methods supporting supplier selection. European Journal of Purchasing and Supply Management, 7(2): 75-89.

[6] Degraeve Z, Labro E \& Roodhooft F. 2000. An evaluation of supplier selection methods from a Total Cost of Ownership perspective. European Journal of Operational Research, 125(1): 34-59.

[7] DICKSON GW. 1966. An analysis of vendor selection systems and decisions. Journal of Purchasing, 2(1): 5-17.

[8] EDWARDS W \& BARRON FH. 1994. SMARTS and SMARTER: Improved simple methods for multiattribute utility measurement. Organizational Behavior and Human Decision Processes, 60(3): 306-325.

[9] EdWARds W. 1977. How to use multiattribute utility measurement for social decision making. IEEE Transactions on Systems, Man, and Cybernetics, 7(5): 326-340.

[10] EHTAмо H \& HÄмÄLÄINEN RP. 2001. Interactive multiple-criteria methods for reaching Pareto optimal agreements in negotiations. Group Decision and Negotiation, 10(6): 475-491.

[11] FISHER R. 1978. International Mediation: A Working Guide, New York: International Peace Academy.

[12] GUo X \& LiM J. 2007. Negotiation support systems and team negotiations: the coalition formation perspective. Information and Software Technology, 49: 1121-1127.

[13] HA SH \& KRISHNAN R. 2008. A hybrid approach to supplier selection for the maintenance of a competitive supply chain. Expert Systems with Applications, 34: 1303-1311. 
[14] Ho W, XU X \& DEY PK. 2010. Multi-criteria decision making approaches for supplier evaluation and selection: A literature review. European Journal of Operational Research, 202(1): 16-24.

[15] Holsapple CW, Lai H \& Whinston AB. 1998. A formal basis for negotiation support system research. Group Decision and Negotiation, 7: 203-227.

[16] Holt GD. 1998. Which contractor selection methodology? International Journal of Project Management, 16(3): 153-164.

[17] Jiao J, You X \& Kumar A. 2006. An agent-based framework for collaborative negotiation in the global manufacturing supply chain network. Robotics and Computer-Integrated Manufacturing, 22(3): 239-255.

[18] Keeney RL \& Raiffa H. 1976. Decisions with multiple objectives: Preferences and value tradeoffs. New York: Wiley.

[19] Klein M, Faratin P, Sayama H \& Bar-Yam Y. 2003. Negotiating complex contracts. Group Decision and Negotiation, 12(2): 111-125.

[20] Lai G, Li C, Sycara K \& Giampapa J. 2004. Literature review of multi-attribute negotiations. Technical Report, CMU-RI-TR-04-66, Carnegie Mellon University, Pittsburgh, USA.

[21] LAi GM, Li CH \& SyCARA K. 2006. Efficient multi-attribute negotiation with incomplete information. Group Decision and Negotiation, 15(5): 511-528.

[22] Lim LH \& BenBASAT I. 1993. A theoretical perspective of negotiation support systems. Journal of Management Information System, 9(3): 27-44.

[23] Lin RJ \& ChOU ST. 2004. Mediating a bilateral multi-issue negotiation. Electronic Commerce Research and Applications, 3: 126-138.

[24] Lomuscio A, Wooldridge M \& Jennings NR. 2003. A classification scheme for negotiation in electronic commerce. Group Decision and Negotiation, 12(1): 31-56.

[25] López-Carmona MA, Marsá-Maestre I \& Velasco JR. 2009. Constraint Based Automated Multi-attribute Negotiations. In: Multiagent Systems [edited by AHMED S \& KARSITI MN], I-Tech, $21-54$.

[26] Louta M, Roussaki I \& Pechlivanos L. 2008. An intelligent agent negotiation strategy in the electronic marketplace environment. European Journal of Operational Research, 187(3): 1327-1345.

[27] Luo X, Jennings NR, Shadbolt N, Leung H \& Lee JH. 2003. A fuzzy constraint based model for bilateral multi-issue negotiations in semi-competitive environments. Artificial Intelligence, 148(1-2): 53-102.

[28] NASH JF. 1950. The Bargaining Problem. Econometrica, 18(2): 155-162.

[29] RaIfFA H. 1982. The art and science of negotiation: How to resolve conflicts and get the best out of bargaining. Cambridge: The Belknap Press of Harvard University Press.

[30] Sandholm T, Sikka S \& Norden S. 1999. Algorithms for optimizing leveled commitment contracts. In: Proceedings of the $16^{\text {th }}$ International Joint Conference on Artificial Intelligence, Stockholm, Sweden, 535-540.

[31] SAndholm T. 2000. Agents in electronic commerce: Component technologies for automated negotiation and coalition formation. Autonomous Agents and Multi-Agent Systems, 3(1): 73-96. 
[32] Schmidt AS, Jobim Filho H, Jobim MSS, Silva VM, Gobus L, Scapin J \& Bieger F. 2001. Integração das cadeias produtivas: Sistema de avaliação de fornecedores de materiais e componentes na indústria da construção civil. Relatório de Pesquisa, Universidade Federal de Santa Maria, Santa Maria, Brasil.

[33] Schramm F \& Morais DC. 2012. Decision support model for selecting and evaluating suppliers in the construction industry. Pesquisa Operacional, 32(3): 643-662.

[34] Szajubok NK, Mota CMM \& Almeida AT. 2006. Uso do método multicritério ELECTRE TRI para classificação de estoques na construção civil. Pesquisa Operacional, 26(3): 625-648.

[35] Verma R \& Pullman ME. 1998. An analysis of the supplier selection process. Omega, 26(6): 739-750.

[36] Vincke P. 1992. Multi-criteria decision aid. Bruxelles: John Wiley and Sons.

[37] WANG J \& ZIONTS S. 2008. Negotiating wisely: Considerations based on MCDM/MAUT. European Journal of Operational Research, 188: 191-205.

[38] Weber CA, Current JR \& Benton WC. 1991. Vendor selection criteria and methods. European Journal of Operational Research, 50(1): 2-18.

[39] Wu DD, Zhang Y, Wu D \& OLson DL. 2010. Fuzzy multi-objective programming for supplier selection and risk modeling: A possibility approach. European Journal of Operational Research, 200(3): 774-787.

[40] Zhang L, Song H, Chen X \& Hong L. 2011. A simultaneous multi-issue negotiation through autonomous agents. European Journal of Operational Research, 210: 95-105. 